\title{
Shared Control for Wheelchair Using Fuzzy Logic
}

\author{
Bruna Mendes Barbosa*. Leonardo Rocha Olivi*. Exuperry Barros Costa*. \\ *Faculdade de Engenharia, Universidade Federal de Juiz de Fora, \\ Rua José Lourenço Kelmer, s/n - Campus Universitário - Bairro São Pedro - CEP: 36036-900 \\ Juiz de Fora-MG-Brasil (Tel: (32)2102-3911 ; e-mail: bruna.barbosa@engenharia.ufjf.br).
}

\begin{abstract}
The medical area in conjunction with engineering have developed techniques that can create Human-Machine Interfaces, facilitating the interaction between equipment and people with disabilities. The use of mobile robots associated with assistive technologies can restore the mobility of these people. These interfaces have inherent classification errors and thus require shared controls between the machine and the human in order to supervise navigation and compensate for the classification errors inherent in those interfaces. In this article we propose the shared control of a wheelchair using fuzzy logic, capable of adapting to any type of environment, making navigation safe, accurate and free of collision risks.
\end{abstract}

Resumo: A área médica em conjunto com a engenharia tem desenvolvido técnicas que podem criar Interfaces Humano-Máquina, facilitando a interação entre equipamentos e pessoas portadoras de deficiências. $\mathrm{O}$ uso de robôs móveis associados a tecnologias assistivas podem recuperar a mobilidade dessas pessoas. Essas interfaces possuem erros de classificação inerentes, e, assim, necessitam-se de controles compartilhados entre a máquina e o humano, de modo a supervisionar a navegação e compensar os erros de classificação inerentes a essas interfaces. Neste artigo é proposto o controle compartilhado de uma cadeira de rodas utilizando lógica fuzzy, capaz de se adaptar a quaisquer tipos de ambientes, possibilitando uma navegação, segura, acurada e livre de riscos de colisão.

Keywords: fuzzy systems; shared control; assistive robotics; wheelchair control; mobile robotics.

Palavras-chaves: Sistemas fuzzy; controle compartilhado; robótica assistiva; controle de cadeiras de rodas; robótica móvel.

\section{INTRODUÇÃO}

Com o passar das descobertas e pesquisas, a robótica torna-se cada vez mais presente em nossa realidade. Uma das áreas com maior crescimento tem sido na área da saúde, com mais de 5 mil cirurgias robotizadas já realizadas no Brasil, que atende em hospitais particulares e pelo Sistema Único de Saúde (Exame, 2018; SUS, 2019). As aplicações desse tipo de tecnologia crescem qualitativa e quantitativamente e criam novas possibilidades para pessoas portadoras de deficiências físicas, como tetraplegia, esclerose múltipla, acidentes vasculares, dentre outros exemplos. (Olivi et al., 2013).

De acordo com o censo do Instituto Brasileiro de Geografia e Estatística (IBGE, 2010), 23,9\% da população brasileira, atualmente 50,02 milhões de pessoas) tem algum tipo de deficiência, seja ela visual, motora, cognitiva, dentre outras. O mesmo censo aponta que a privação motora atinge $7 \%$ da população, aproximadamente 14,65 milhões de indivíduos. Tratam-se de percentuais elevados, mostrando que uma parcela significativa da população demanda cuidados.

Nos casos envolvendo deficiência motora, a recuperação da mobilidade é um grande desafio e o foco principal deste trabalho. Existem casos em que se pode restabelece-la por meio de procedimentos médicos e fisioterápicos. No entanto, para outras situações mais graves, o indivíduo fica confinado permanentemente numa cadeira de rodas.
Ainda assim, é possível o uso de outras tecnologias para auxiliar na recuperação da mobilidade. Dentre as diversas tecnologias desenvolvidas para a área médica e com possibilidades de utilização na robótica, enquadram-se a eletromiografia (EMG) e a eletroencefalografia (EEG), que são tecnologias desenvolvidas para aquisição de sinais biológicos, e usadas em interfaces de comunicação com usuários que tem suas habilidades motoras restritas (Sanei \& Chambers, 2007).

A EMG é uma tecnologia útil para extrair sinais biológicos de pacientes, seja ele facial ou em outras partes do corpo. Uma característica desta tecnologia é a possibilidade de seu uso de maneira não-invasiva. Entretanto, em razão do uso não-invasivo, perde-se acurácia na obtenção das informações em decorrência dos ruídos e atenuações de sinal. Assim, são imperativas medidas mitigadoras quanto aos erros de leituras dos sinais biológicos dos usuários (Sanei \& Chambers, 2007).

De acordo com Emotiv (2019), o equipamento utilizado neste trabalho, para mapear a verdadeira atividade do cérebro e músculos, é muito importante medir os sinais de diversos locais diferentes. Sendo assim, um dos grandes desafios nestes casos é o tratamento que será dado aos erros de leitura no equipamento, uma vez que essas interfaces apresentam erros inerentes na identificação da ação. A Figura 1 exibe os equipamentos de uma aplicação de cadeiras de rodas robóticas. 


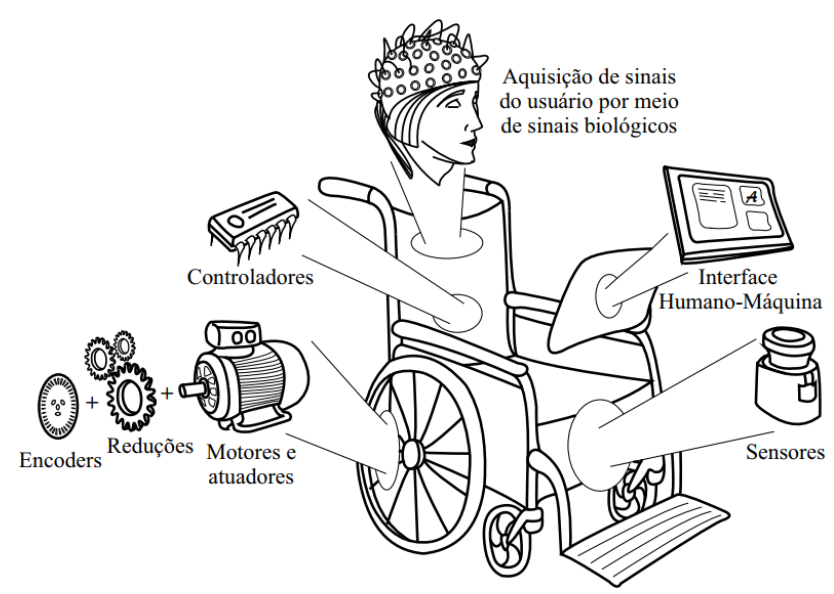

Fig. 1 Arquitetura do sistema robótico (Olivi et al., 2013).

Tendo em vista que as interfaces de comunicação humanomáquina que utilizam sinais biológicos são afetadas por ruídos e distorções aleatórias, prejudicando a extração de sinais do usuário de um sistema robótico assistivo, neste trabalho será proposto um controle compartilhado para cadeiras de rodas robóticas utilizando a lógica fuzzy (Pedrycz, \& Gomide, 2007) para minimizar as possíveis situações de risco e maximizar a mobilidade e segurança durante a navegação, devolvendo ao mesmo, em parte, sua mobilidade.

\section{TRABALHOS CORRELATOS}

O conceito de controle compartilhado está diretamente relacionado a divisão da responsabilidade entre o usuário e uma lei de controle na atuação e direcionamento de um sistema robótico (Stoelen et al., 2012). Uma lei de controle assistiva deve ajudar o usuário a completar uma tarefa de controle de um robô de modo a maximizar a segurança do usuário enquanto a tarefa é realizada (Olivi et al., 2013).

Conforme a Figura 2, o usuário dispõe de uma interface humano-máquina assistiva baseada em Brain Computer Interface (BCI), capaz de extrair os comandos adquiridos diretamente do usuário, os quais são enviados ao robô (Liu et al., 2011; Olivi et al. 2013). O sistema compartilhado leva, portanto, as decisões do usuário em consideração, no entanto, se propõem a avaliar se houve algum problema na extração dos comandos do usuário por parte da Interface HumanoMaquina, filtrando os possíveis erros de classificação.

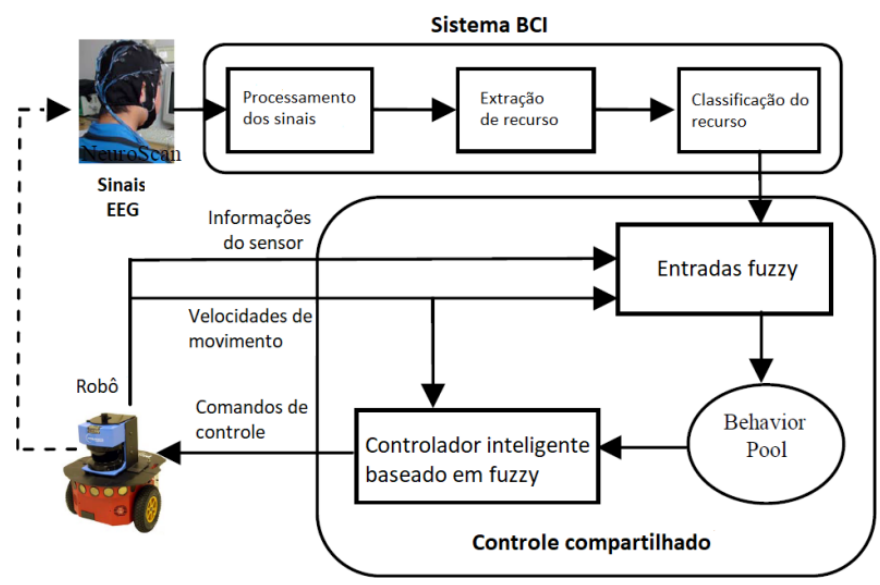

Fig. 2 Arquitetura do sistema robótico com EEG/EMG.
Portanto, além das leituras do robô e das informações do ambiente, ainda é possível ao controlador basear na percepção e direcionamento do usuário.

Os autores de Liu et al. (2011) demonstraram o uso de um controle compartilhado entre a coleta de sinais através do EEG e as informações lidas pelos sensores do robô utilizado no experimento. De acordo com os autores, os principais problemas da interface entre o computador e o cérebro estão associados ao baixo número de canais e ao alto erro na leitura dos sinais. Os autores utilizam um método de controle de movimento assistivo para que os impulsos que comandam o robô sejam confiáveis e em tempo real. Portanto, para que o robô responda com maior eficácia ao ambiente externo, um controle compartilhado baseado em lógica fuzzy foi utilizado.

O trabalho de Olivi et al. (2013) desenvolveu um controle compartilhado baseado em campos vetoriais aplicado para cadeiras de rodas robóticas. Os autores descrevem as diversas metodologias de controle compartilhado, dentre as quais a maioria utiliza situações padronizadas cujas configurações não atendem aos diversos ambientes. Os autores apresentam, então, uma metodologia capaz de se adaptar a quaisquer tipos de ambientes, mostrando diversas situações problemáticas em que o robô é capaz de se adaptar à disposição de obstáculos do ambiente e calcular as melhores velocidades para a cadeira de rodas robótica de maneira a auxiliar o usuário a concluir sua navegação pelos diversos cômodos maximizando a segurança do robô, evitando colisões e vencendo os obstáculos mais comuns presentes na rotina de um ambiente.

O trabalho apresentado por Nishimura et al. (2015) descreve um sistema de controle compartilhado utilizado para dirigir um veículo automotivo. O principal objetivo do artigo foi encontrar uma interação suave entre o objetivo do motorista e o objetivo do sistema automatizado do veículo. Foi estudada a possibilidade de apoiar o condutor para que acompanhasse uma faixa automotiva ao mesmo tempo que possibilitasse que o mesmo mudasse de faixa, conforme a intenção do condutor.

Desta maneira, este trabalho se propõe a elaborar um controle compartilhado para cadeiras de rodas robóticas baseado em lógica fuzzy que seja adaptável a diversos ambientes, uma vez definida a distância de proteção, que minimize os erros de leitura da interface humano-máquina e maximize a segurança do usuário, ajudando-o a completar a navegação com liberdade de movimentos.

\section{METODOLOGIA PROPOSTA}

A principal comunicação de um indivíduo com deficiência, a qual impede a manipulação de interfaces de interação convencionais como joysticks, mouses e teclados, é por meio de seus sinais biológicos. O sistema nervoso é o responsável por produzir sinais elétricos através de atividades eletroquímicas complexas, que serão capazes de controlar as funções do corpo humano, tais como a movimentação voluntária. Temos então a eletromiografia, que será apresentada neste trabalho como forma de extração dos sinais biológicos do usuário de modo a construir uma via de comunicação com a máquina. Para tanto, será utilizado o equipamento Emotiv EPOC (Emotiv, 2019) via EMG para guiar um robô móvel por meio de ambientes. 
Propõe-se o uso do controle compartilhado, que atuará na divisão de responsabilidade entre o usuário e uma lei de controle na atuação e direcionamento de um sistema robótico.

Conforme mostrado na Figura 3 o usuário irá dispor de uma interface humano-máquina assistiva capaz de extrair comandos adquiridos diretamente do usuário, e os enviar ao robô móvel. Dessa forma, o principal objetivo do controle compartilhado seria propiciar proteção ao usuário durante a navegação, além de executar correções e minimizar erros da Interface Humano-Máquina Assistiva, atuando diretamente nas velocidades do robô móvel.

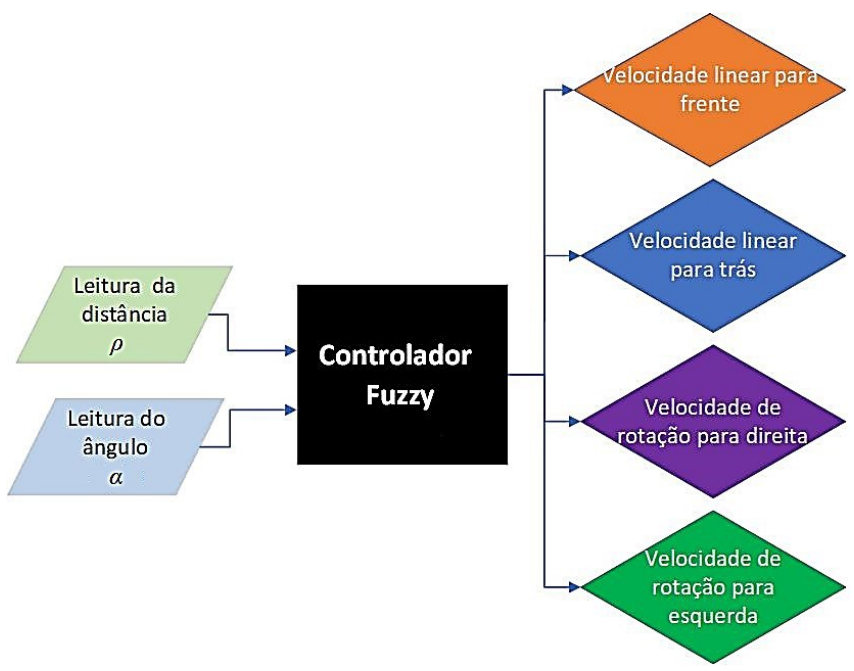

Fig. 3 Esquema das entradas e saídas do controlador fuzzy.

Para definir as funções de pertinência de um sistema fuzzy, é necessário, primeiramente, modelar as variáveis de entrada e saída. Conforme a Figura 3, serão utilizadas duas entradas: i) $\rho$, a distância Euclidiana entre o estado atual do robô e os obstáculos presentes no ambiente, e ii) $\alpha$, os ângulos que os obstáculos presentes no ambiente se encontram com relação ao sistema de coordenadas do robô. Assim:

$\rho_{i}=\sqrt{\left(x_{o}^{\{i\}}\right)^{2}+\left(y_{o}^{\{i\}}\right)^{2}}$

$\alpha_{i}=a \tan 2\left(y_{o}^{\{i\}}, x_{o}^{\{i\}}\right)$

em que ( $x o\{i\}, y o\{i\})$ são as coordenadas de cada obstáculo $i$ existente na cena com relação aos sistemas de coordenadas do robô, conforme definido na Figura 4.

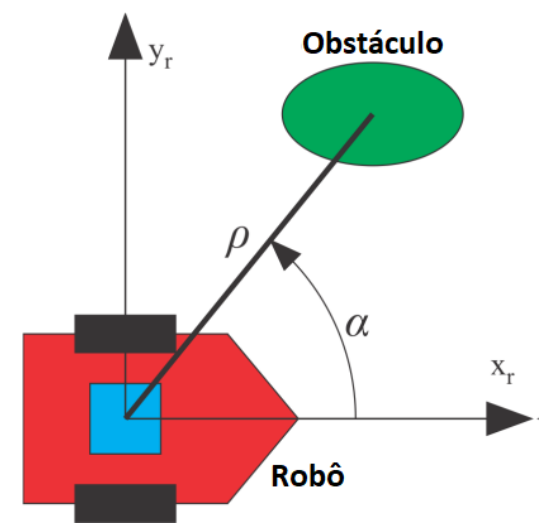

Fig. 4 Variáveis de entrada $\rho$ e $\alpha$.
As saídas do sistema serão velocidades lineares e angulares a serem inseridas diretamente no robô, para moldar sua navegação de acordo com os comandos dados pelo usuário encarregado da cadeira de roda robótica. As saídas são i) velocidade linear para frente, ii) velocidade linear para trás, iii) velocidade de rotação para a direita, e, iv) velocidade de rotação para a esquerda. Com as entradas e saídas definidas, é possível, portanto, modelar as funções de pertinência conforme o comportamento desejado para o robô.

\subsection{Entradas do controlador}

Para a variável $\rho$ decidiu-se de maneira empírica que a distância máxima de influência de um obstáculo sobre o robô será $\rho_{\max }$ de 4 metros, pois esta distância atendeu a todos os ambientes internos testados. Assim, definiu-se de maneira linguística a proximidade do robô aos obstáculos por meio dos conjuntos fuzzy "Muito Perto" (MP), "Perto" (P), "Médio" (M) e "Longe" (L), definidos na Figura 5.

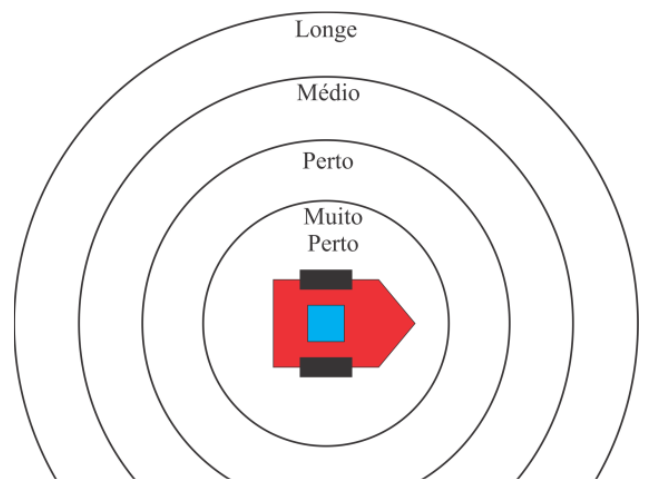

Fig. 5 Definição das regiões definidas para $\rho$.

Primeiramente, tem-se o caso do grupo "Muito Perto", com grau de pertinência de $100 \%$ entre os valores de $0 \mathrm{~m}$ a $0,25 \mathrm{~m}$, decrescendo linearmente entre os valores $0,25 \mathrm{~m}$ e $1,00 \mathrm{~m}$, criando o número fuzzy trapezoidal [0 $\left.\begin{array}{llll}0 & 0 & 0,25 & 1\end{array}\right]$. Para o caso "Perto", tem-se o número fuzzy trapezoidal [0,25 11,25 2]. O mesmo raciocínio é válido para os demais casos, em que o grupo "Médio" é dado pelo número fuzzy [1,25 233 3,75] e "Longe" por [3 3,75 4 4]. Na Figura 6 tem-se, de forma qualitativa, a disposição as regiões consideradas, evidenciando cada um dos grupos fuzzy dados pelos números modelados.

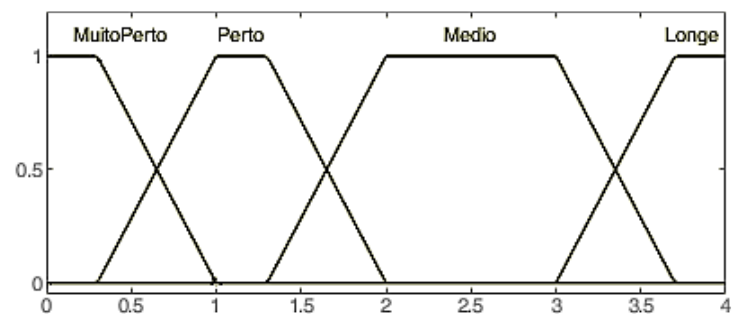

Fig. 6 Entradas $\rho$ e seus grupos do controlador fuzzy.

Para a variável $\alpha$ são considerados cinco grupos fuzzy referentes às regiões do robô, a saber, "Direita Traseira" (DT), "Direita" (D), "Frente" (F), "Esquerda" (E) e "Esquerda Traseira" (ET). Tais regiões estão mostradas na Figura 7, compreendendo os lados físicos do robô. 


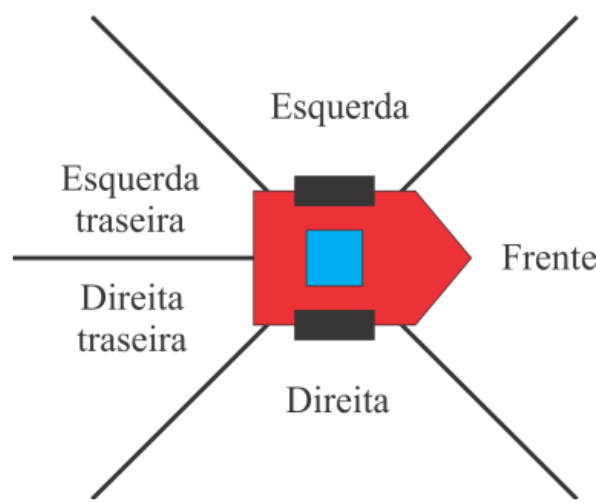

Fig. 7 Definição das regiões definidas para $\alpha$.

Desta maneira, sempre que um obstáculo se apresentar no entorno do robô, é possível classifica-lo em uma dessas cinco regiões de $\alpha$. Assim, o sistema pode decidir quais são as regiões ameaçadas pela proximidade de obstáculos e calcular a reação apropriada. Portanto, conforme mostrado na Figura 8, tem-se os graus de pertinência dos grupos fuzzy "Direita Traseira" $\left[-180^{\circ}-180^{\circ}-135^{\circ}-125^{\circ}\right]$, "Direita $\left[-145^{\circ}-135^{\circ}-\right.$ $\left.45^{\circ}-35^{\circ}\right]$, "Frente" $\left[-55^{\circ}-45^{\circ} 45^{\circ} 55^{\circ}\right]$, "Esquerda" [35 $35^{\circ}$ $\left.135^{\circ} 145^{\circ}\right]$ e "Esquerda Traseira" $\left[125^{\circ} 135^{\circ} 180^{\circ} 180^{\circ}\right]$.

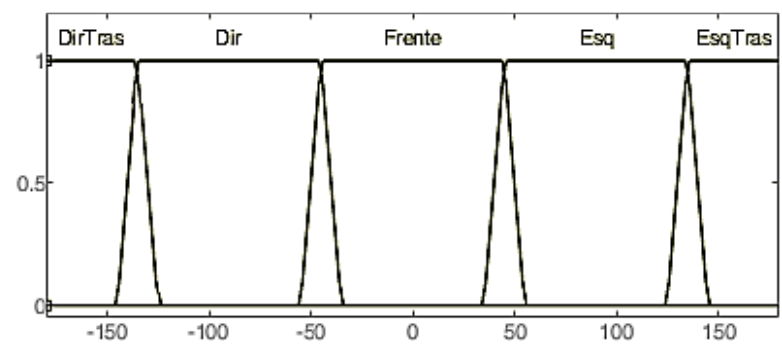

Fig. 8 Entrada $\alpha$ e seus grupos do controlador fuzzy.

\subsection{Saídas do controlador}

Conforme mencionado, as quatro saídas do controlador fuzzy serão: velocidades lineares para frente e para trás, velocidades de rotação para direita e para esquerda. Essas velocidades serão obtidas por meio de quatro fatores de escada contidos no intervalo [0,1], a saber: i) af para velocidade para frente, ii) ar para a velocidade para trás (ré), iii) $a e$ para rotação à esquerda, e iv) $a d$ para rotação à direita, calculados pelo sistema fuzzy. Esses fatores serão, escalas para a velocidades linear e angular máximas permitidas ao robô. Dessa maneira, o sistema fuzzy produz quatro velocidades, duas lineares (frente e trás) e duas angulares (esquerda e direita), independentes e descorrelacionadas entre si, ligadas ao contexto dos obstáculos que se apresentam no ambiente.

Assim, cinco grupos fuzzy foram projetados: "Zero" (Z) [$\left.\begin{array}{lll}0.01 & 0 & 0.01\end{array}\right]$, "Muito Baixo" (MB) [ 0.010 .20 .4$]$, "Baixo" (B) [ 0.2 0.4 0.6], "Médio" (A) [ $\left.\begin{array}{llll}0.4 & 0.6 & 0.8\end{array}\right]$ e "Alto" (A) [0.6 $\left.\begin{array}{lll}0.8 & 1 & 1\end{array}\right]$, e todos os quatro fatores de escala possuem os mesmos grupos. A Figura 9 mostra a função de pertinência utilizada para todos os quatro fatores que produzem as velocidades do robô. Todos são tratados da mesma maneira para que o robô tenha um comportamento padronizado e previsível para o usuário.
O controlador fuzzy recebe as entradas $\rho$ e $\alpha$ do ambiente e deve determinar, com base no contexto dado, quais velocidades são as mais adequadas para que o robô possa navegar de maneira a maximizar a segurança do usuário. Assim, uma base de regras fuzzy relaciona as variáveis de saída às de entrada conforme o contexto do ambiente.

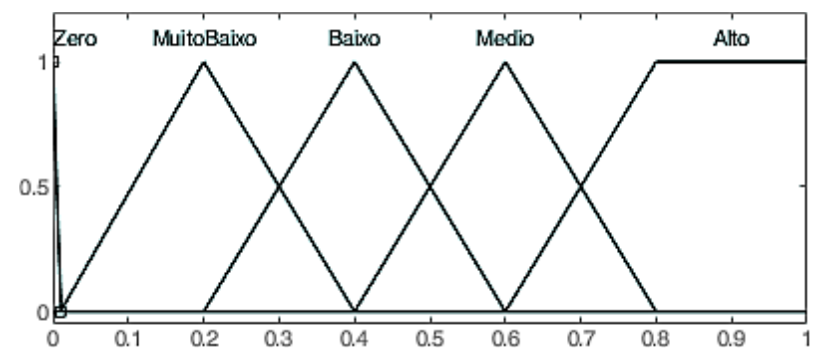

Fig. 9 Perfil dos fatores de escala que produzem a velocidade de saída do robô.

\subsection{Base de regras}

O conjunto de regras do sistema fuzzy descrito possui um conjunto de 20 regras que se devem a combinação das cinco possíveis entradas de $\rho$ com as quatro possíveis entradas de $\alpha$. As superfícies de controle geradas pelas regras para cada um dos termos de saída estão dispostas na Figura 10, mostrando as situações para as quais cada saída é determinada, conforme a entrada recebida. Tem-se, assim, um comportamento não-linear.

Seguem as regras do controlador compartilhado:

1. Se $\rho=M P$ e $\alpha=D T,\{a f=B, a r=Z, a e=M, a d=M B\}$

2. Se $\rho=M P$ e $\alpha=D,\{a f=M B, a r=M B, a e=M, a d=M B\}$

3. Se $\rho=M P$ e $\alpha=F,\{a f=Z, a r=B, a e=M, a d=M\}$

4. Se $\rho=M P$ e $\alpha=E,\{a f=M B, a r=M B, a e=M B, a d=M\}$

5. Se $\rho=M P$ e $\alpha=E T,\{a f=B, a r=Z, a e=M B, a d=M\}$

6. Se $\rho=P$ e $\alpha=D T,\{a f=M, a r=M B, a e=M, a d=B\}$

7. Se $\rho=P$ e $\alpha=D,\{a f=M, a r=M, a e=M, a d=B\}$

8. Se $\rho=P$ e $\alpha=F,\{a f=M B, a r=M, a e=M, a d=M\}$

9. Se $\rho=P$ e $\alpha=E,\{a f=M$, ar $=M, a e=B, a d=M\}$

10. Se $\rho=P$ e $\alpha=E T,\{a f=M, a r=M B, a e=B, a d=B\}$

11. Se $\rho=M$ e $\alpha=D T,\{a f=A, a r=B, a e=M, a d=M\}$

12. Se $\rho=M$ e $\alpha=D,\{a f=A, a r=A, a e=M, a d=B\}$

13. Se $\rho=M$ e $\alpha=F,\{a f=B, a r=A, a e=M, a d=M\}$

14. Se $\rho=M$ e $\alpha=E,\{a f=A, a r=A, a e=B, a d=M\}$

15. Se $\rho=M$ e $\alpha=E T,\{a f=A, a r=B, a e=M, a d=M\}$

16. Se $\rho=L$ e $\alpha=D T,\{a f=A, a r=M, a e=A, a d=A\}$

17. Se $\rho=L$ e $\alpha=D,\{a f=A, a r=A, a e=A, a d=M\}$

18. Se $\rho=L$ e $\alpha=F,\{a f=M$, ar $=A, a e=A, a d=A\}$

19. Se $\rho=L$ e $\alpha=E\{a f=A, a r=A, a e=M, a d=A\}$

20. Se $\rho=L$ e $\alpha=E T,\{a f=A, a r=M, a e=A, a d=A\}$

As regras definidas garantem que para casos onde o obstáculo se encontra muito perto, a velocidade associada àquela direção deva ser nula para evitar a colisão. Nos casos onde o obstáculo está perto, ainda é permitida uma velocidade muito baixa na direção obstruída enquanto nas demais direções associadas a velocidade é baixa, tudo isto para tornar a navegação segura sem tolher a liberdade do usuário de escolher qualquer direção. 

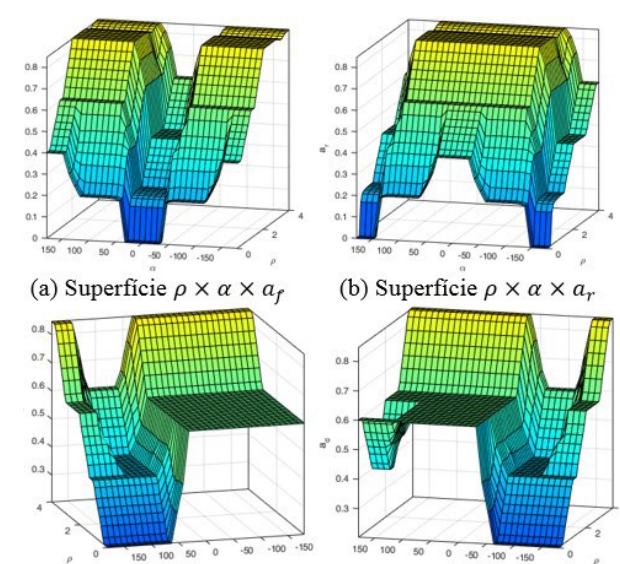

(b) Superfície $\rho \times \alpha \times a_{r}$

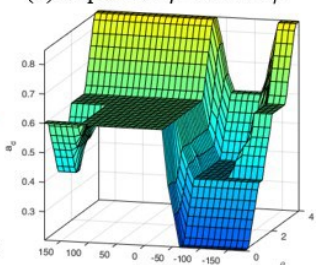

(d) Superfície $\rho \times \alpha \times a_{d}$

Fig. 10 Superfícies de controle: a) $a f$, b) $a r$, c) $a e$, e d) $a d$.

\section{RESULTADOS}

Esta seção apresentará os resultados da avaliação do controlador desenvolvido por meio de simulação utilizando o Mobile SIM e o robô Pioneer P3-DX, com o Emotiv (2019) no modo Hardware in The Loop (HIL).

\subsection{Testes isolados nas regras fuzzy}

Para ilustrar os resultados apresentados pelo controle compartilhado, pode-se primeiramente avaliar casos isolados para que seja possível identificar a forma como os gráficos de entrada e saída do controlador irão atuar. Na Figura 11 tem-se a navegação com uma parede à esquerda, e nas Figuras 12 e 13 tem-se o comportamento das saídas fuzzy do controlador ao navegar próximo a uma parede à esquerda e à direita.

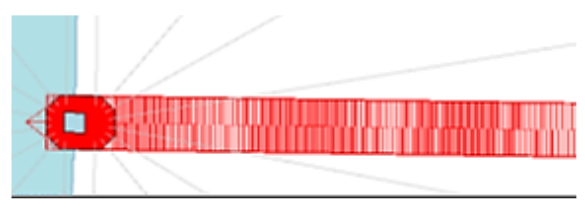

Fig. 11 Navegação com parede à esquerda do robô.
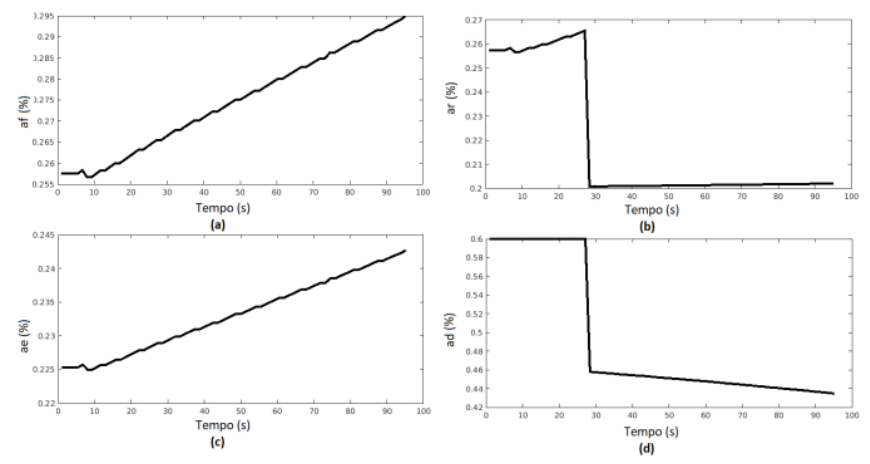

Fig. 12 Saídas do controlador com parede a esquerda do robô, para a) $a f$, b) $a r$, c) $a e$, e d) $a d$.

Por meio das Figuras 11 e 13, é possível visualizar a ação individual do robô para cada situação, mostrando que o planejamento das regras fuzzy alcançaram seus objetivos. A seguir, coloca-se o robô em situações mais desafiadoras, presentes no dia comum de qualquer ambiente interno.
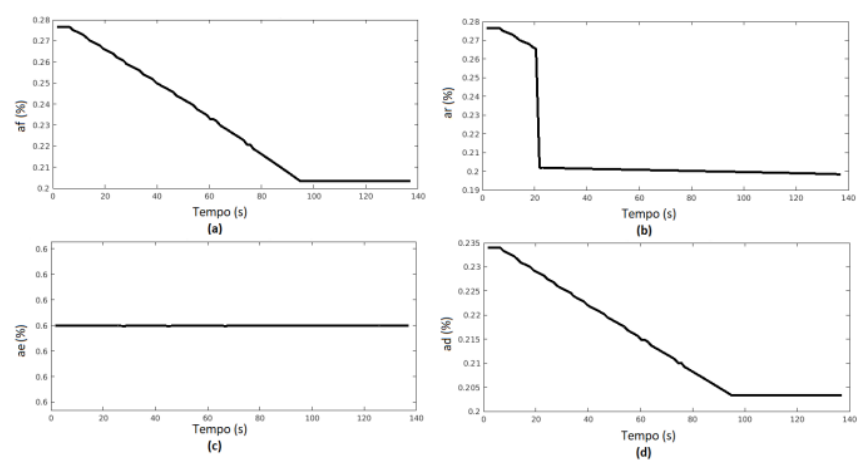

Fig. 13 Saídas do controlador, com o passar do tempo, com parede a direita do robô, para a) $a f$, b) $a r$, c) $a e$, e d) $a d$.

\subsection{Navegação por corredores estreitos}

Corredores estreitos são desafios recorrentes na vida de qualquer cadeirante. Controlar um robô móvel por meio de uma Interface Humano-Máquina que produzem erros de classificação torna-se ainda mais desafiador.

A Figuras 14 mostra a navegação por um corredor bastante estreito com relação às proporções do robô. Durante 223,7 segundos de navegação, o robô percorreu uma distância de 5,36 metros, com uma velocidade média de 24 milímetro/s, usual na literatura. A navegação ocorreu com segurança, nenhuma colisão, o usuário conseguiu manter o robô no centro do corredor por todo o trajeto, que é o local mais seguro possível, com o auxílio do controle compartilhado, mesmo em face a diversos erros de classificação do Emotiv.

Desta maneira, é possível observar o quanto este controle compartilhado maximizou a segurança do usuário e minimizou os erros de classificação do Emotiv. A Figura 15 mostra o perfil de velocidades linear e angular do robô por todo o trajeto, condizentes com os desafios.

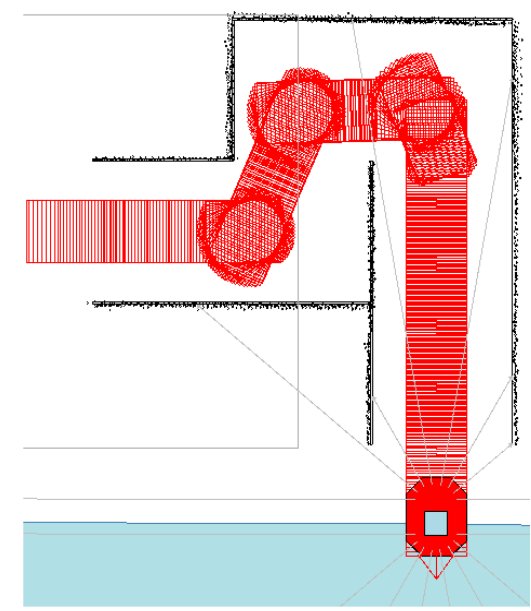

Fig. 14 Navegação por um corredor estreito.

\subsection{Navegação por longas distâncias}

O último experimento mostra a navegação do robô por ambientes internos maiores, com passagens de corredores, portas, e toda gama de desafios para um cadeirante. 

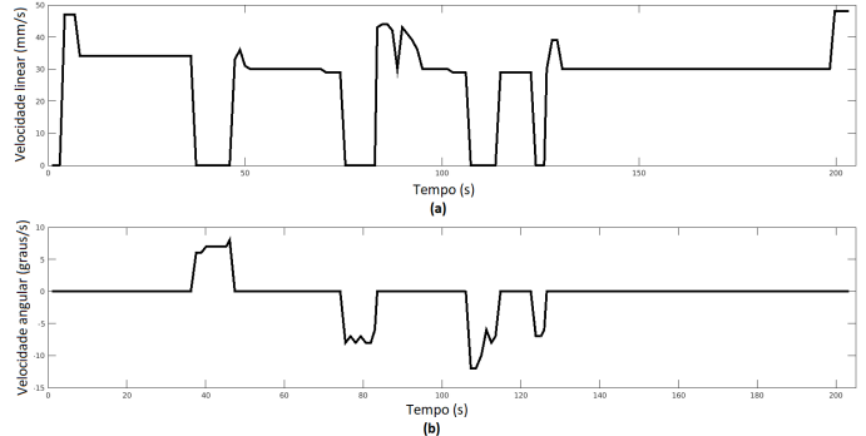

Fig. 15 Perfil de velocidades (a) linear, em $\mathrm{mm} / \mathrm{s}$ e (b) angular, em graus/s, para o trajeto do corredor estreito.

O desempenho do usuário com controle compartilhado pode ser observado nas Figuras 15 e 16, em que diversas dificuldades se impuseram ao usuário, que, por meio do Emotiv e do controle compartilhado, pôde vencê-las, recuperando sua autonomia e mobilidade pelo ambiente.

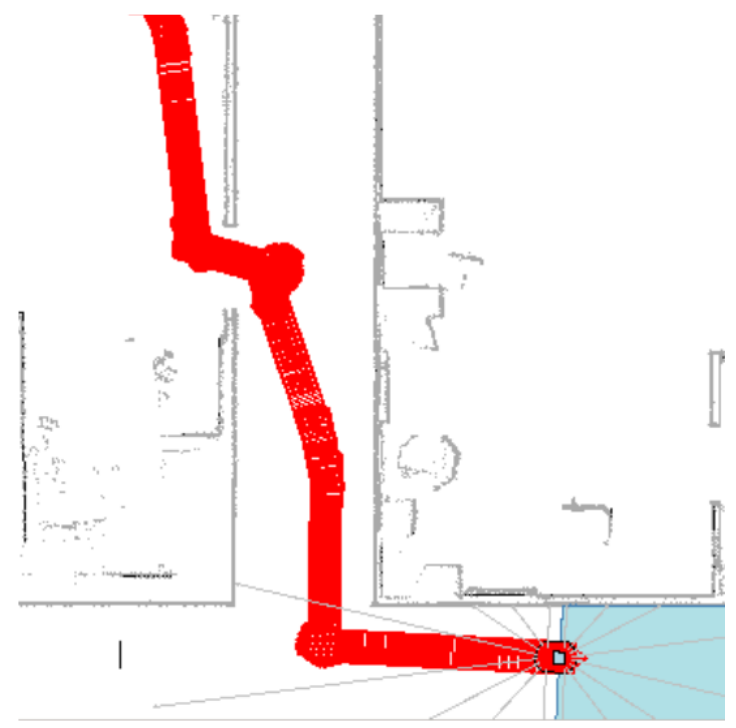

Fig. 16 Navegação por longas distâncias.

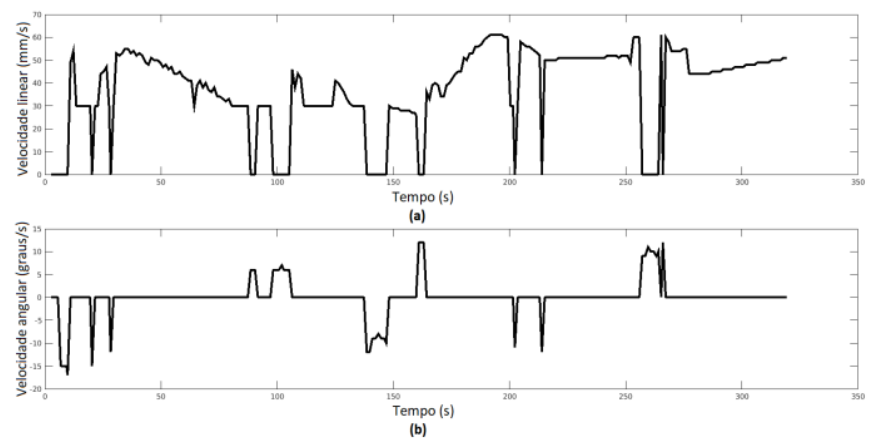

Fig. 17 Perfil de velocidades (a) linear e (b) angular.

\section{CONCLUSÕES}

Este trabalho apresentou um controlador fuzzy no contexto de controle compartilhado para cadeiras de roda robóticas, tornando a navegação do usuário inclusiva com relação aos seus comandos e vontades, acurada e livre de riscos de colisão, mesmo com diversos obstáculos e dificuldades encontradas ao longo dos percursos mais comuns em ambientes internos.

Os resultados mostraram que o desenvolvimento técnico utilizado obteve sucesso em todas as situações para as quais foi exposto, nunca permitindo que o usuário fosse colocado em situações de colisão, minimizando todos os erros de classificação do sistema de BCI Emotiv, mantendo o usuário sempre em uma situação de segurança e liberdade. Mesmo em comparação com os resultados obtidos na literatura, o controlador aqui desenvolvido demonstrou excelente desempenho, sendo competitivo e computacionalmente leve.

Como sugestões de trabalhos futuros, é possível que a base de regras seja ampliada, se tornando ainda mais robusta quanto ao controle compartilhado. Portanto, aumentar o número de regras de forma a contemplar ainda mais possibilidades aos ambientes nos quais o robô está inserido.

\section{REFERÊNCIAS}

Emotiv (2019). The Science behind our technology. Disponível em: https://www.emotiv.com/the-science/. Acessado em 05/07/2018.

Exame, Revista (2018). Aumenta o número de cirurgias robóticas no Brasil. Disponível em: $<$ http://exame.abril.com.br/negocios/dino/aumenta-onumero-de-cirurgias-roboticas-no-brasil/>. Acessado em: 05/05/2019.

IBGE, Instituto Brasileiro de Geografia e Estatística (2010). Censo 2010. Disponível em: $<$ http://censo2010.ibge.gov.br/>. Acessado em: 05/05/2019.

Liu, R.; Xue, K.-Z.; Wang, Y.-X.; Yang, L. (2011). A FuzzyBased Shared Controller For Brain-Actuated Simulated Robotic System. IEEE Engineering in Medicine and Biology Society.

Nishimura, R.; Wada, T.; Sugiyama, S. (2015). Haptic Shared Control In Steering Operation Based On Cooperative Status Between A Driver And A Driver Assistance System. Journal of Human-Robot Interaction, v. 4, n. 3, p. 19-37.

Olivi, L.; Souza, R.; Rohmer, E.; Cardozo, E. (2013). Shared control for assistive mobile robots based on vector fields. The IEEE 10th International Conference on Ubiquitous Robots and Ambient Intelligence (URAI 2013), South Korea.

Pedrycz, W.; Gomide, F. (2007). Fuzzy Systems Engineering: Toward Human-Centric Computing. Editora WileyBlackwell.

Sanei, S.; Chambers, J.A. (2007). EEG Signal Processing. Ed. Wiley.

Stoelen, M.F.; Tejada, V.F.; Huete, A.J.; Bonsignorio, F.; Balaguer, C. (2012). Benchmarking Shared Control for Assistive Manipulators - From Controllability to the SpeedAccuracy Trade-Off. IEEE/RSJ International Conference on Intelligent Robots and Systems.

SUS, Sistema Único de Saúde (2019). Disponível em: $<$ http://portalms.saude.gov.br/sistema-unico-de-saude $>$. Acessado em: 05/05/2019. 